for an $R C$ filter and

$$
f_{c}=\left(10^{11}\right) / 5.5=18 \mathrm{GHz}
$$

for a Gaussian filter.

Therefore, information in the $3-\mathrm{MHz}$ to $18-\mathrm{GHz}$ frequency band is distorted using the $R C$ filter to eliminate aliasing, but it is not distorted using the Gaussian filter.

The particular filters chosen were used for illustrative purposes only and do not represent the complete set of efficient antialiasing filters. However, this example illustrates the need for a careful choice of filter to reduce the effects of aliasing when using sampled time-domain information to generate frequency-domain information.

\section{REFERENCES}

[1] W. D. McCaa, Jr. and N. S. Nahman, "Generation of reference waveforms by uniform lossy transmission lines," IEEE Trans. Instrum. Meas., vol. IM-19, pp. 382-390, Nov. 1970.

[2] R. A. Lawton, N. S. Nahman, and J. M. Bigelow, "A solid state reference waveform standard," IEEE Trans. Instrum. Meas., vol. IM-33, pp. 201-205, Sept. 1984.

\title{
Mode-Stirred Chamber Calibration Factor, Relaxation Time, and Scaling Laws
}

\author{
ROBERT E. RICHARDSON, JR.
}

\begin{abstract}
In this paper, single-mode cavity theory relating cavity $Q$, stored energy, and relaxation time is applied to mode-stirred chamber (MSC) operation.

It is shown that a calibration factor for average power density, defined in terms of stored energy density, can be determined solely from a relaxation time measurement, for which a suitable measurement technique is also shown.

A possible definition of minimum operating frequency for an MSC is also defined in terms of parameters that can easily be measured during normal chamber operation.

Finally, an attempt is made to show how the derived and measured results apply to $\mathrm{MSC}$ operation and design.
\end{abstract}

\section{Calibration Factor Definition in Terms of $Q$ and Relaxation Time}

$\mathrm{T}$ HE calibration factor (denoted here as $M$ ) for a modestirred chamber (MSC) is generally given by a relationship of the form:

$$
M=\frac{\langle P D\rangle_{\mathrm{eff}}}{P_{T}} \quad(\mathrm{~cm})^{-2}
$$

where $\langle P D\rangle_{\text {eff }}$ is the "effective" power density in the chamber and $P_{T}$ is the net transmitter power into the chamber. For a free-space plane wave, power density which is customarily given by the Poynting Vector, may also be given as the product of light speed $c$ and the energy

\footnotetext{
Manuscript received March 22, 1985; revised July 17, 1985. This work was supported by NSWC Independent Exploratory Development (IED) Funding.

The author is with the Naval Surface Weapons Center, Dahlgren, VA.
}

density $u$ :

$$
\begin{aligned}
P D=\frac{1}{2} \operatorname{Re} \overline{E \times H^{*}} & =c\left(\frac{\epsilon_{0} E^{2}}{2}+\frac{\mu_{0} H^{2}}{2}\right) \\
& =c u \quad\left(\mathrm{w} / \mathrm{cm}^{2}\right) .
\end{aligned}
$$

For the discussion of MSC operation in this paper we define $\langle P D\rangle_{\text {eff }}$ as the product of light speed and average energy density in an MSC, or

$$
\langle P D\rangle_{\mathrm{eff}}=c \bar{u} \quad\left(\mathrm{w} / \mathrm{cm}^{2}\right) .
$$

The average energy density may be measured to within any desired accuracy by using a suitable number of calibrated $E$ or $H$ field probes, having minimal perturbation.

The $Q$ of a cavity may be written generally as the nondimensional parameter

$$
Q=\omega \frac{\text { Stored Energy }}{P_{T}}
$$

where $P_{T}$ is net input power to the cavity, and $\omega$ the radian frequency. By measuring the average stored energy in the Test MSC and interpreting $Q$ as a composite [1] $Q$, (3) and (4) may be combined with (1) and (2) to yield

$$
M=\frac{\langle P D\rangle_{\mathrm{eff}}}{P_{T}}=\frac{c \bar{u}}{P_{T}}=\frac{c Q}{\omega V} \quad\left(\mathrm{~cm}^{-2}\right)
$$

where $V$ is the chamber volume.

Furthermore, the $1 / e$ decay time $(\tau)$ of energy stored in a cavity mode may be written as [2] 


$$
\tau=\frac{Q}{\omega} \quad(\mathrm{sec}) .
$$

Interpreting (6) as the decay time for a particular "average" mode in an MSC we may write:

$$
M=\frac{\langle P D\rangle_{\mathrm{eff}}}{P_{T}}=\frac{c \bar{u}}{P_{T}}=\frac{c Q}{\omega V}=\frac{c \tau}{V}(\mathrm{~cm})^{-2} .
$$

Equation (7) is important for two reasons. First, as is to be discussed further in this paper, it shows that the chamber calibration factor, as shown in (3), may be evaluated by two independent means; either (a) a direct, frequencydomain measurement of $\langle P D\rangle_{\text {eff }}$ and $P_{T}$ with field probes and power meters, or (b) a decay time measurement requiring a linear (but not absolutely calibrated) detector and a suitably designed sampling system with an accurate time base. In principle it appears that the decay time measurement might be more accurate since time interval can be measured accurately with greater ease than microwave power density. Second, (7) shows that for applications where an MSC is to be used to generate or measure a pulsed EM environment, there would be an upper limit to the maximum allowable calibration factor unless some additional correction factor were to be applied to allow for the chamber ringing, which limits the minimum allowable pulsewidth.

\section{Time-Domain ApProach}

An alternative derivation of $\tau$ may be conducted utilizing a "time-domain" approach, rather than the frequencydomain approach. First, the number $(n)$ of passes or reflections a wave (ray) makes in order to decay to $1 / e$ of its initial value is calculated:

$$
|\Gamma|^{2^{n}}=\frac{1}{e}
$$

where $|\Gamma|^{2}$ is the (power) reflection coefficient for an EM wave reflected from a metallic surface.

Then

$$
\tau=\frac{n l}{c}(\mathrm{sec})
$$

where $\eta$ is a characteristic chamber dimension $\left(V^{1 / 3}\right)$ and $c$ is light speed. Equation (8) can be evaluated by using the relation

$$
\operatorname{Ln}(1+x) \simeq x, \quad|x| \ll 1 .
$$

Also the refraction coefficient $\Gamma$ is generally given as

$$
\Gamma \simeq 1-\frac{2 Z_{m}}{2 s}=1-2 \sqrt{\frac{j \omega \epsilon_{0} \hat{\mu}}{\sigma}}
$$

and

$$
\Gamma * \Gamma=|\Gamma|^{2}=1-2 \sqrt{2} \sqrt{\frac{\omega \hat{\mu} \epsilon_{0}}{\sigma}}
$$

where $Z_{m}$ is the impedance of the metallic conducting wall and $Z_{0}$ is free-space impedance. The microscopic quan- tities have their usual significance, in particular, $\hat{\mu}$ is the relative permeability of the wall material which would be unity for Aluminum or Copper and greater than unity or perhaps even complex and nonlinear for iron.

Evaluation of (8) using (10) leads to:

$$
\begin{aligned}
n \operatorname{Ln}|\Gamma|^{2} & =\operatorname{Ln} \frac{1}{e}=-1 \\
-\frac{1}{n} & =-2 \sqrt{2} \sqrt{\frac{\omega \hat{\mu} \epsilon_{0}}{\sigma}} \\
n & =\frac{1}{2 \sqrt{2}} \sqrt{\frac{\sigma}{\omega \hat{\mu} \epsilon_{0}}}
\end{aligned}
$$

and

$$
\tau=\frac{l}{2 \sqrt{2}} \sqrt{\frac{\sigma \mu_{0}}{\omega \hat{\mu}}} .
$$

The quantity $M$ is then given simply by

$$
M=\frac{c \tau}{V}=\frac{1}{2 \sqrt{2}} \frac{1}{V^{2 / 3}} \sqrt{\frac{\sigma}{\omega \hat{\mu} \epsilon_{0}}} .
$$

The calibration factor $M$ as it appears in (15) is nearly equal to the equation which would be derived by evaluation of $M$ as ${ }^{1}$

$$
M=\frac{c Q}{\omega V} \quad\left(\mathrm{~cm}^{-2}\right)
$$

\section{Transient Response Analysis Technique}

For purposes of analysis it is assumed that the MSC transient response consists of a sum of exponentially decaying sinusoids of the form

$$
V_{0}=\sum_{i} a_{i} \sin \left(\omega_{i} t+\theta_{i}\right) e^{-t / 2 \tau_{i}}
$$

where the terms $a_{i}, \omega_{i}, \theta_{i}, \tau_{i}$ represent the initial amplitude, frequency, phase angle, and relaxation time for the voltage components received on the MSC reference antenna from the modes present during any given paddle wheel position and excitation frequency range. Each paddle wheel position results in a different function $V_{0}(t)$ with different parameters in (6).

Of particular interest is the value or range of values for $\tau$. We deduce a best fit average value for $\tau$ by forming an ensemble average of $V_{0}(t)^{2}$ behavior which is done by sampling and squaring $V_{0}(t)$ at discrete time, $t$ steps for many different paddle wheel positions. This operation is shown pictorially in Fig. 1.

It can be shown [3] that for a sum of random independent variables $V_{k}$ :

' $Q$ is often given as $Q=3 / 2(V / S \delta)$ a more exact expression is $Q=$ $3 / 2 V /\left(S \sqrt{2 \hat{\mu} / \omega \mu_{0} \sigma}\right)$ which is different for $\hat{\mu} \neq 1$. The approximate expression is likely to be interpreted incorrectly for magnetic materials because relative permeability $(\hat{\mu})$ appears in the numerator in the radical sign rather than in the denominator with $\mu_{0}$. The beauty of the simpler expression is only "skin deep." 


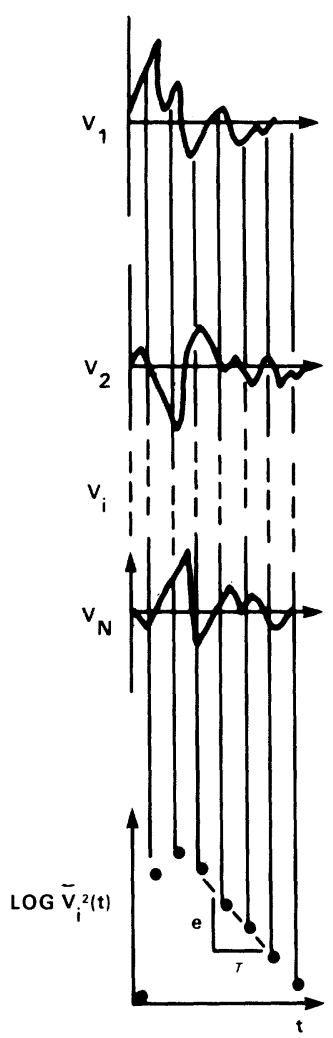

Fig. 1. Ensemble averaging technique for MSC transient response signals.

$$
\overline{\left(\sum_{k} V_{k}\right)^{2}}=\sum_{k} \overline{V_{k}^{2}}
$$

Thus

$$
\begin{aligned}
\sum \overline{V_{0}^{2}} & =\overline{\left(\sum V_{0}\right)^{2}} \\
& =\sum_{k}\left(\sum_{i} a_{i k} \sin \left(\omega_{i k} t+\theta_{i k}\right) e^{-t / 2 \tau_{i k}}\right)^{2} \\
& =\sum_{k} \sum_{i} a_{i k}^{2} \sin ^{2}\left(\omega_{i k} t+\theta_{i k}\right) e^{-t / \tau_{i k}}
\end{aligned}
$$

where the bars represent ensemble (not time) averages. If we assume that $\tau$ values fall within a narrow sharply peaked distribution with an average value $\bar{\tau}$ we may factor out the exponential term of (18):

$$
\overline{V_{0}^{2}}(t) \approx\left(\sum_{k} \sum_{i} a_{i k}^{2} \sin ^{2}\left(\omega_{i k} t+\theta_{i k}\right)\right) e^{-t / \bar{\tau}}
$$

The double sum term inside the brackets in (19) has a stationary average value and $\overline{V_{0}^{2}}(t)$ thus has an exponentially decreasing value with a time constant $\bar{\tau}$ which can be easily deduced from curve fitting a plot of $V_{0}^{2}$ sampled at various times $t$ following the cessation of the MSC excitation pulse.

\section{Measurement Systems for Experimental EVAluation OF $M$}

\section{A. Direct Evaluation}

The measurement system for experimentally evaluating the MSC calibration factor directly is shown in Fig. 2.
Calibrated $E$ and $H$ field probes are sequentially switched to a receiver which yields a dc voltage proportional to $E^{2}$ or $H^{2}$ at each probe. These readings together with incident and reflected power readings at each of many paddle wheel positions are averaged.

It was found to be very convenient to use low perturbation "button" $E$-field probes [4] mounted in the chamber walls. Preliminary data indicate that these readings agree with standard (commercially available) $E$-field probe readings to within 1-3 dB. Such probes are quite simple to build and calibrate. Controversy exists however as to whether field measurements made at MSC walls are representative of the fields inside, because of possible surface waves, and other effects.

\section{Relaxation Time Measurement Scheme}

\section{A. Equipment Setup}

MSC transient reponse was measured using the setup described in Figs. 3 and 4. The MSC is driven by a shortpulsed microwave signal derived from a stable $\mathrm{CW}$ source which also serves as a local oscillator for a mixer where the chamber output is detected.

The mixer output feeds a sampling oscilloscope which displays the detected MSC response, which has been shifted back to the baseband.

The sampling oscilloscope is controlled by a small computer with interfacing $\mathrm{A} / \mathrm{D}$ and $\mathrm{D} / \mathrm{A}$ converters as shown in Fig. 4. The computer generates a counting sequence which generates a "staircase" at the output of the D/A converter. At a particular "staircase" value which tells the oscilloscope the time point at which to sample the transient signal, a dc voltage appears at the oscilloscope output and is $\mathrm{A}$ to $\mathrm{D}$ converted for storage and processing in the computer. Following each "staircase" cycle, which samples the transient signal at between 20-100 points, a paddle wheel stepping motor control pulse is generated by the computer which then begins a new "staircase" sampling cycle on a new transient response resulting from the new paddle wheel position. Generally about 50-400 paddle wheel positions are counted (each with 20-100 points) to form an "ensemble average" transient response estimate.

\section{B. Measurement Results}

Typical detected transient response (appearing at the mixer output) following application of a pulsed microwave signal is shown in Fig. 5. The signal swings both positive and negative and its gradual decrease with time is nonmonotonic.

Fig. 6 is a semi-logarithmic plot of $\overline{V^{2}}(t)$ that can be fitted easily to arrive at a best fit value for $\bar{\tau}$.

In Fig. $7 \overline{V^{2}}(t)$ is plotted on a linear scale showing chamber behavior during, as well as following, application of a pulse. One can easily observe the (averaged) exponential build up of fields to form the standing wave pattern responsible for the chambers power density multiplication effects. 


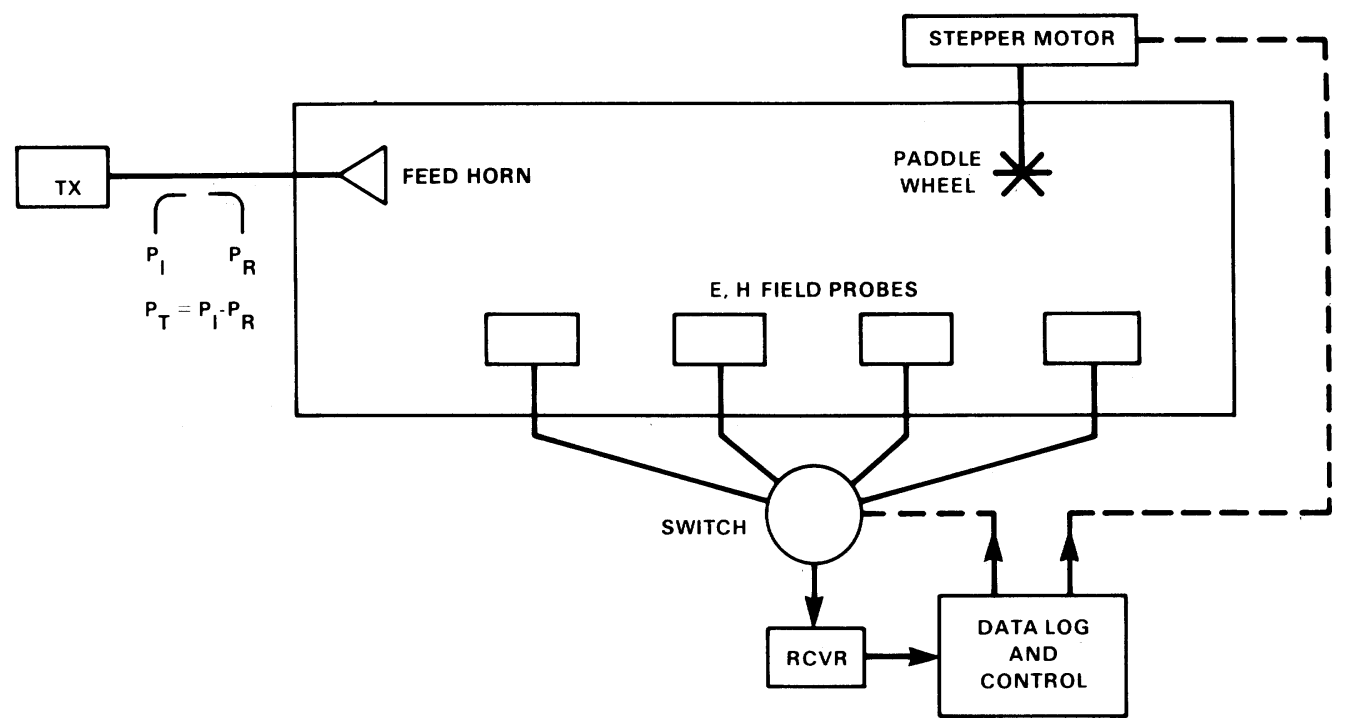

Fig. 2. Assembly for direct measurement of MSC calibration factor.

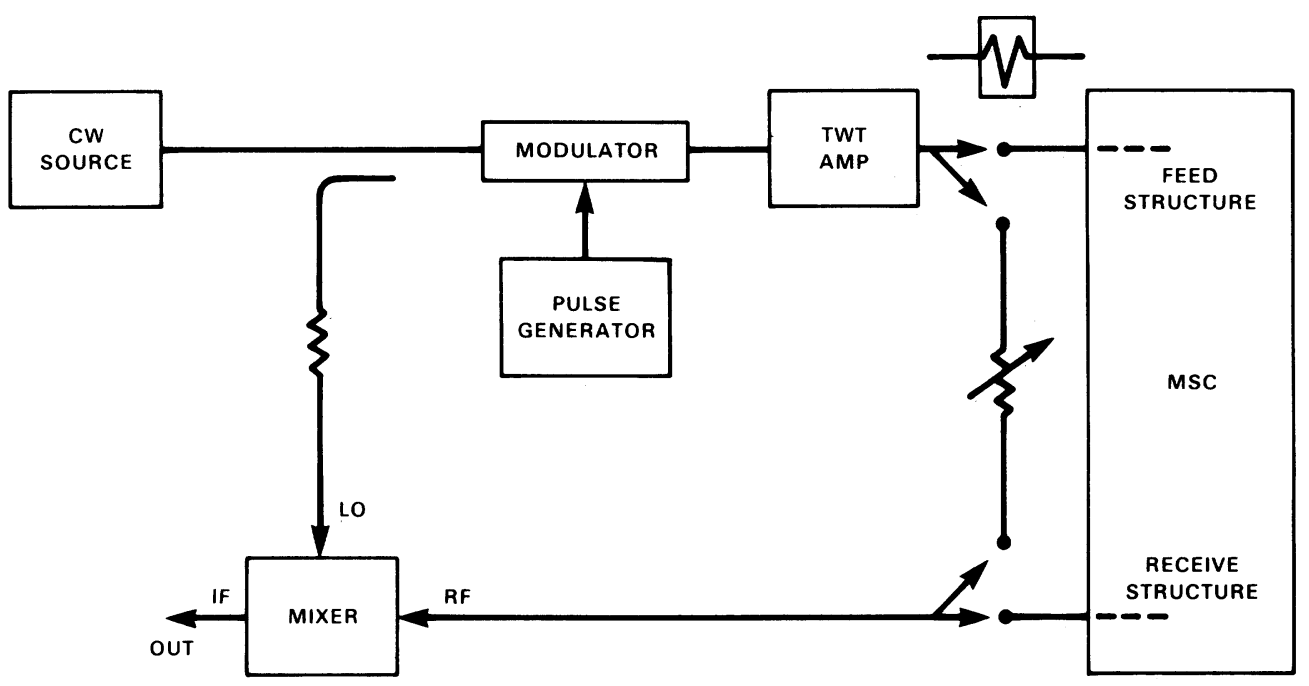

Fig. 3. Schematic of assembly for examining transient response of an MSC.

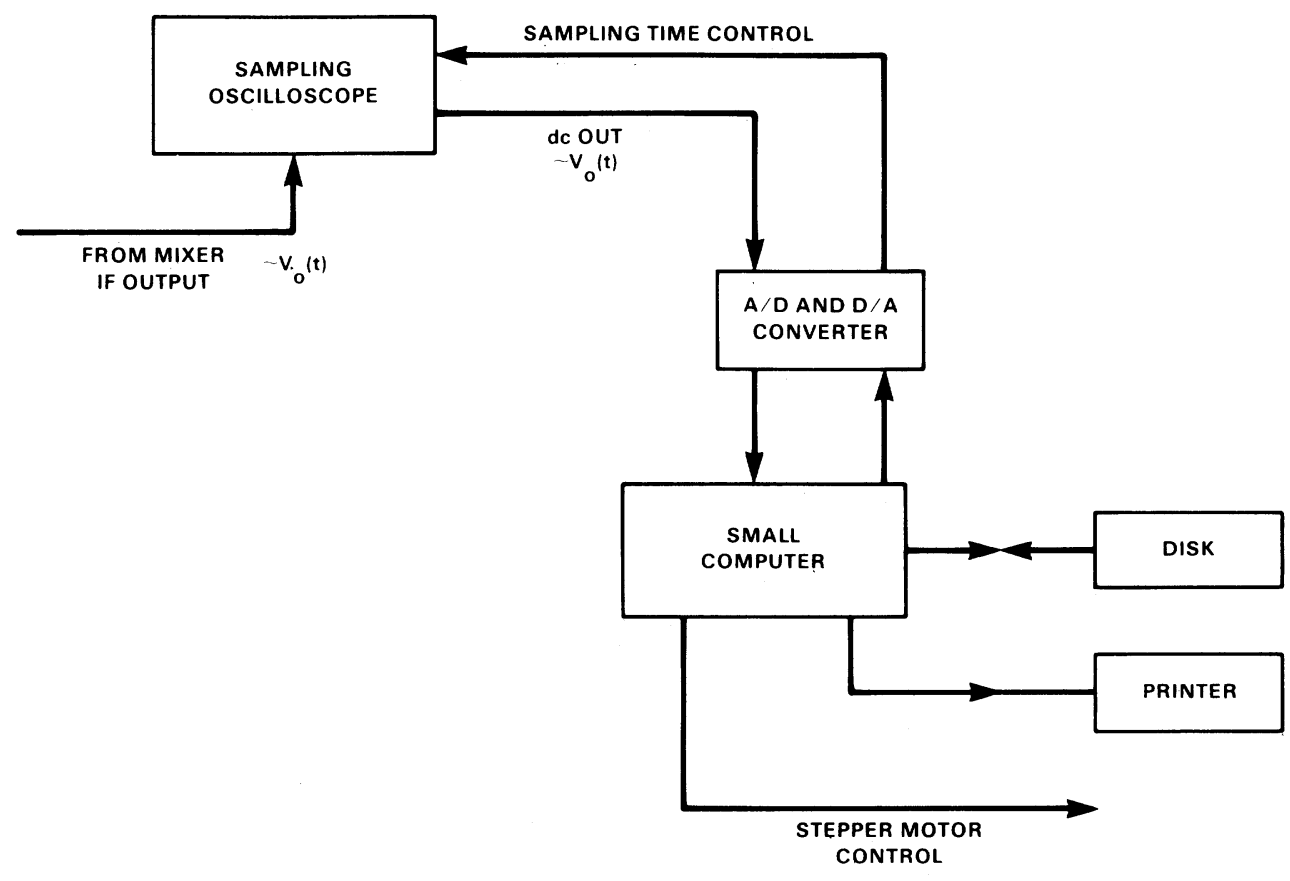

Fig. 4. Data acquịsition from sampling oscilloscope for relaxation time determination. 


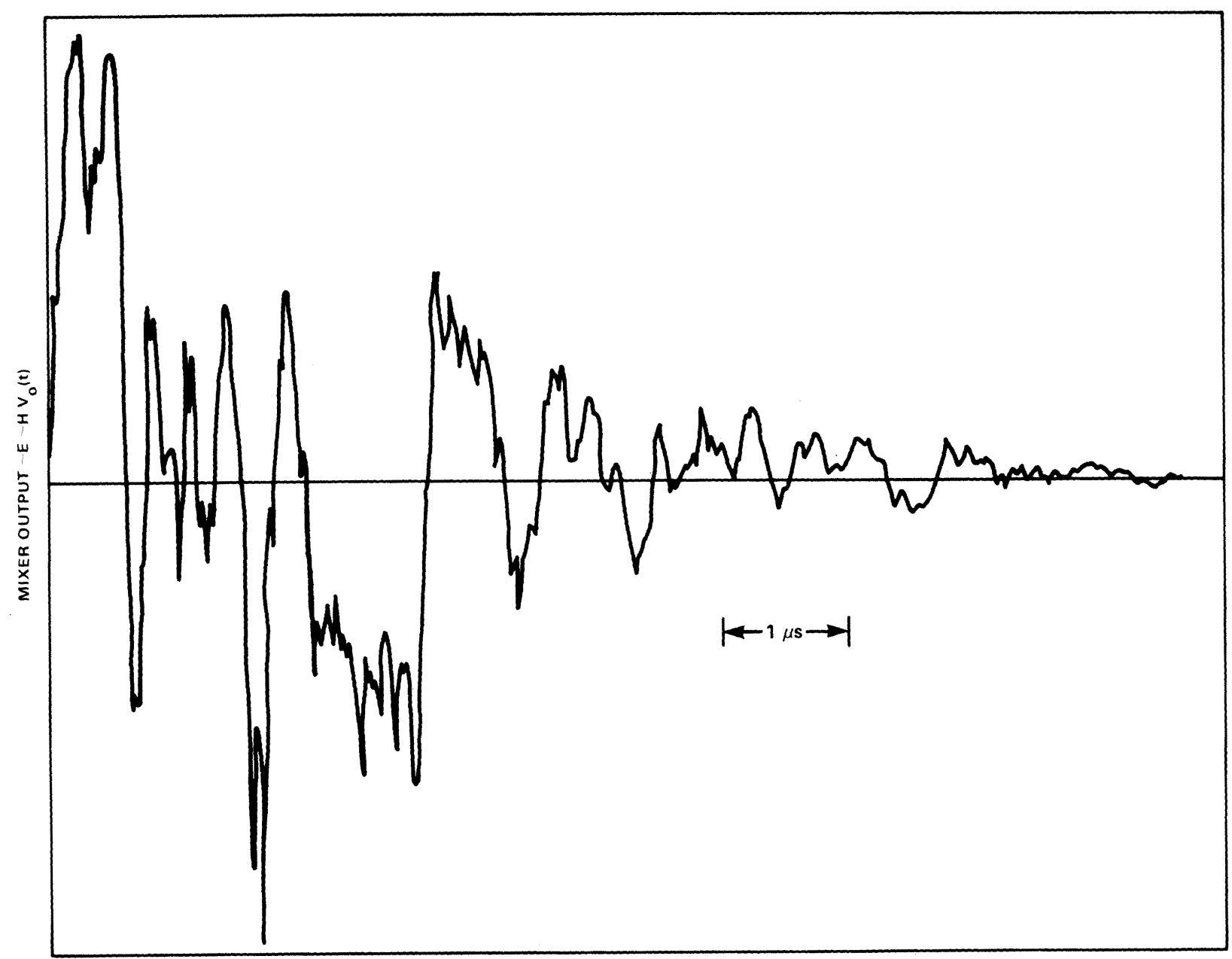

Fig. 5. Typical transient response curve from an MSC as measured at homodyne mixer output.

In Fig. 8 the direct calibration factor as measured with field probes is plotted versus the quantity $(c \tau / V)$ for two chambers in operation at the Naval Surface Weapons Center (NSWC). The large steel-lined chamber was measured both when empty and when loaded with several pieces of absorbent material to show the direct trade off between relaxation time and calibration factor for a particular installation. Measurements on a somewhat smaller copper wall chamber show that it has a significantly higher $M$ value, consistent with the results of (14), (15) (the equations actually predict much larger $M$ values than are observed experimentally).

\section{Spectral Analysis of Transient Response}

The transient response of an MSC can also be evaluated with spectral analysis techniques. Peaks in the ringing response can be examined and compared with predictions from Weyl or other [11] formulations for mode density.

If one connects a spectrum analyzer to the MSC output and applies a pulsed-microwave input, responses as shown in Fig. 9 are obtained. The filtered chamber output contains a number of peaks which may, perhaps, be associated with MSC modes.

"Good" chamber operation is generally assumed to occur where the mode density is high enough so that modes overlap with each other because their line width is greater than their spacing. If one takes the product of mode density

$$
\frac{d N}{d F}=8 \pi V \frac{F^{2}}{c^{3}} \quad(\operatorname{modes} /(\mathrm{Hz})
$$

and mode line width

$$
\Delta F=\frac{1}{2 \pi \tau}
$$

then one can arrive at a measure of mode overlap by the factor $A$ :

$$
A=\frac{4 V F^{2}}{c^{3} \tau} .
$$

Evaluation of (20) with parameters for the Copper test chamber at NSWC yields the plot shown in Fig. 10. Also plotted is measured peak density from analysis of many plots similar to that in Fig. 9 with different carrier frequencies and paddle wheel positions.

Fig. 10 shows that the break in observed spectral peak density from that predicted by (20) occurs at about the same frequency where (22) has unit value.

At lower freqencies where significant mode overlap does 


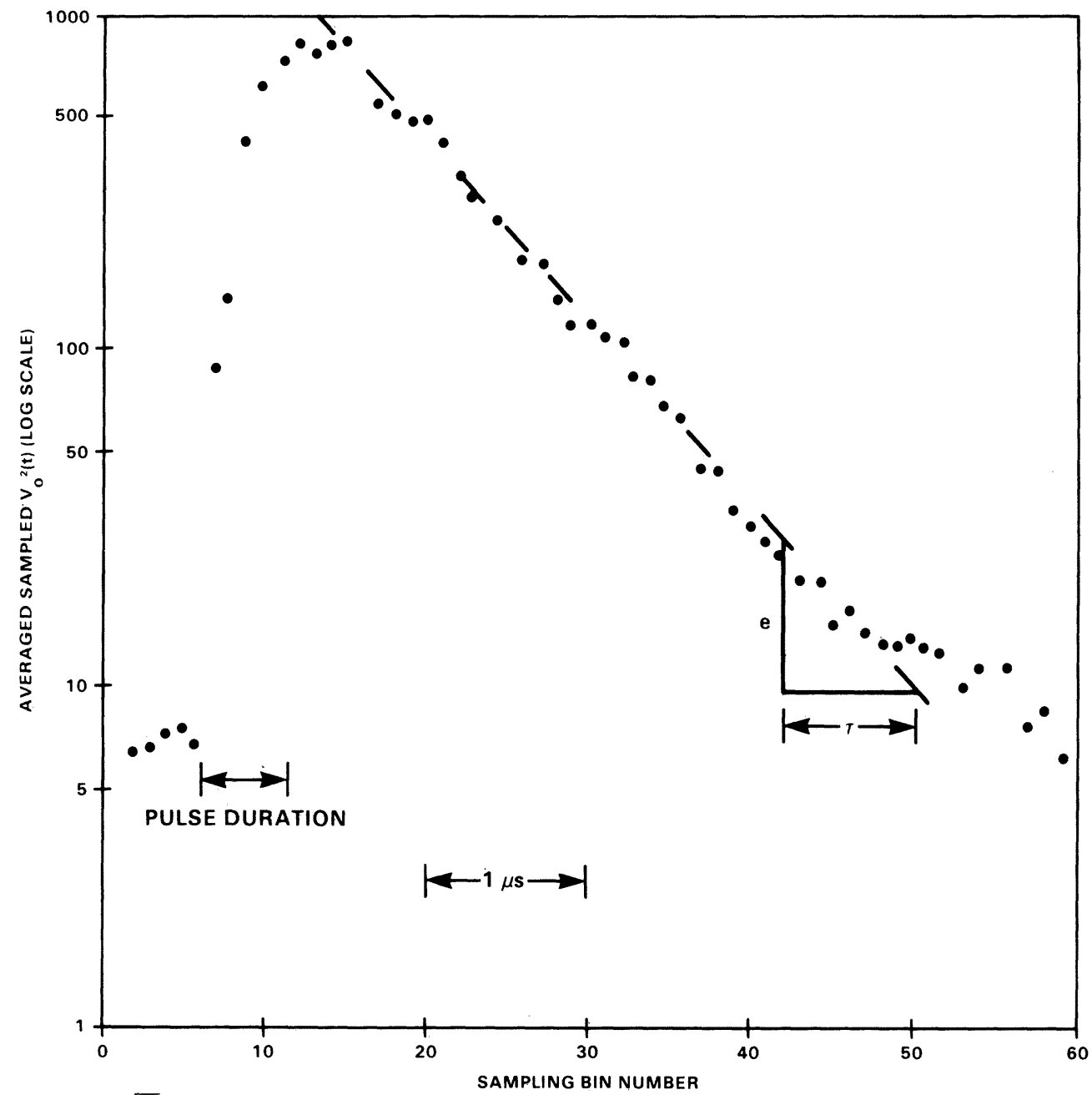

Fig. 6. $\overline{V^{2}}(t)$ versus sampling bin number plotted on semi-logarithmic scale to show experimental decay.

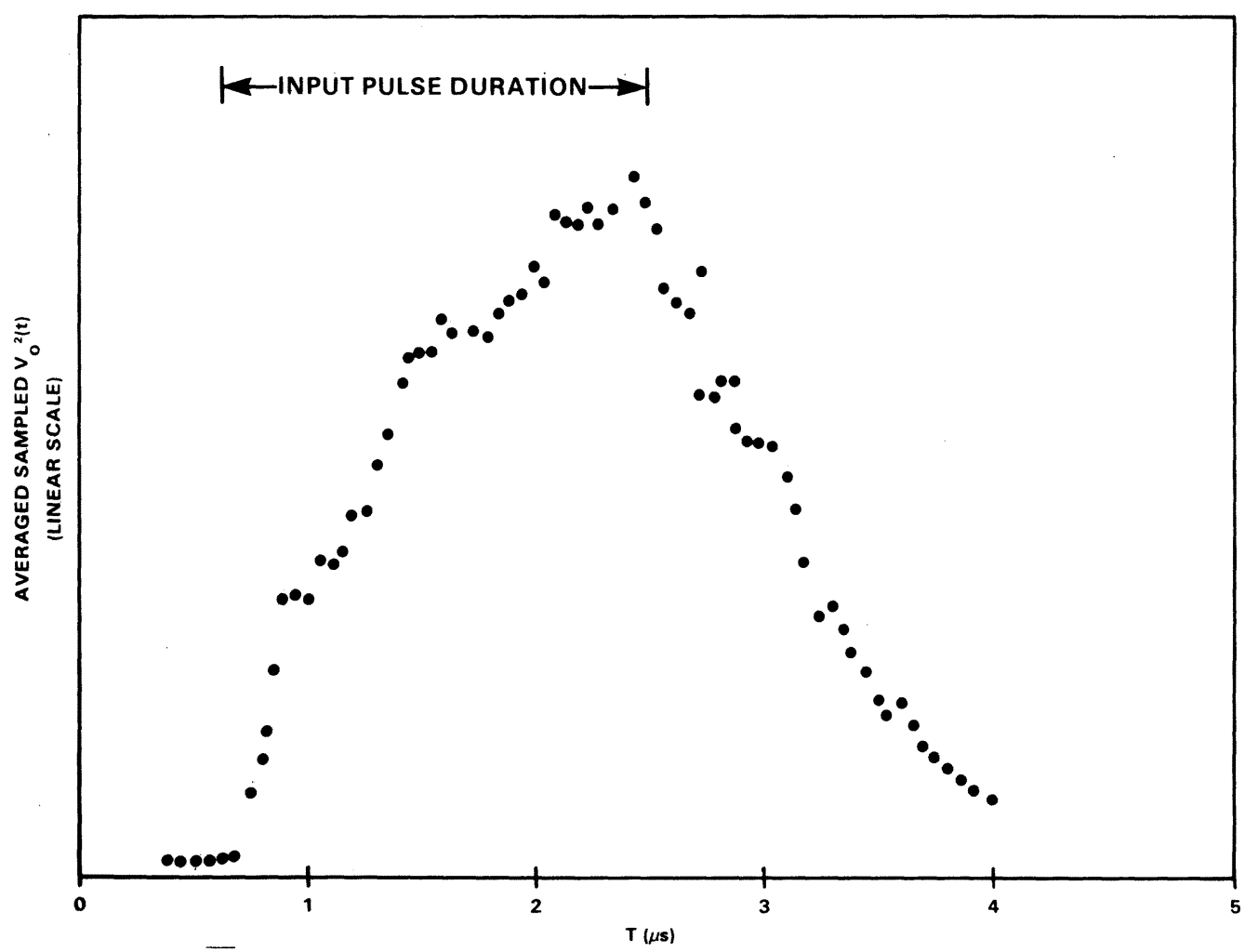

Fig. 7. $\overline{V^{2}}(t)$ versus time plotted on linear scale to show exponential build-up of chamber fields. 


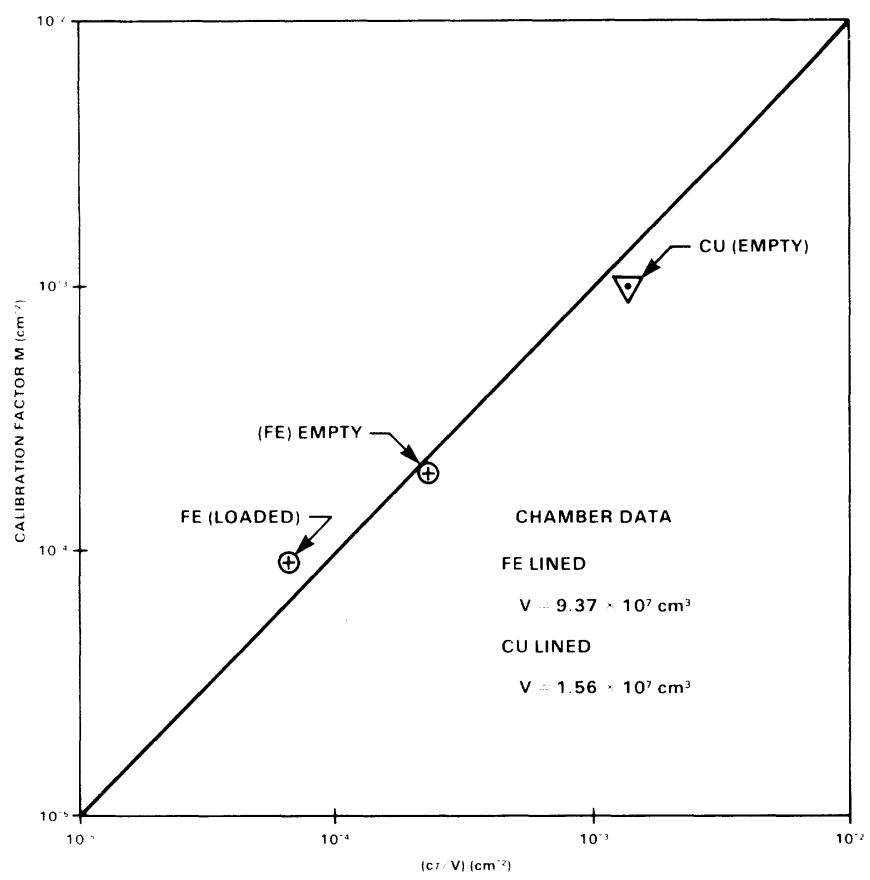

Fig. 8. Directly measured calibration factor plotted versus $(c \tau / V)$ for two mode-stirred chambers, in operation at N.S.W.C.

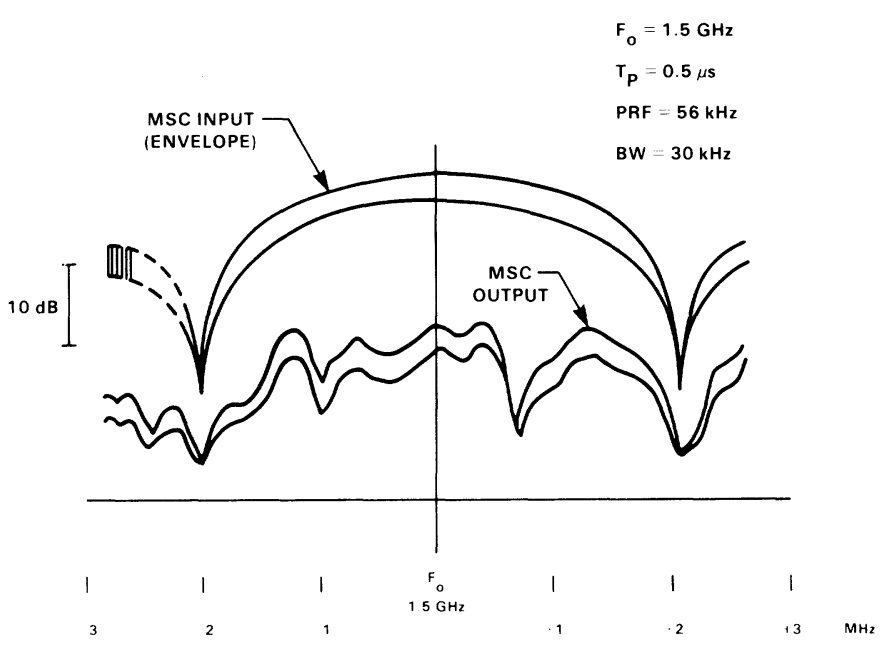

Fig. 9. Measured spectra of MSC input and output signals.

not occur the spectral peaks may correspond to modes. At higher frequencies where significant overlap does occur, i.e., $A \gg 1$, a different interpretation is necessary.

In numerous measurements where investigators have measured power density in different regions of an MSC and also for many different paddle wheel positions, there is observed to exist a ratio of about 7-10 dB between the peak and average power density. This ratio holds nearly constant $^{2}$ even at frequencies significantly above the threshold frequency, where suitable mode overlap is assumed to occur.

Inspection of the data in Fig. 9 shows that a similar ratio exists between peak and average or mean spectral response. Since the peak to average ratio in MSC power density measurements is of considerable interest, it may

${ }^{2} \mathrm{M}$. Crawford, private communication.

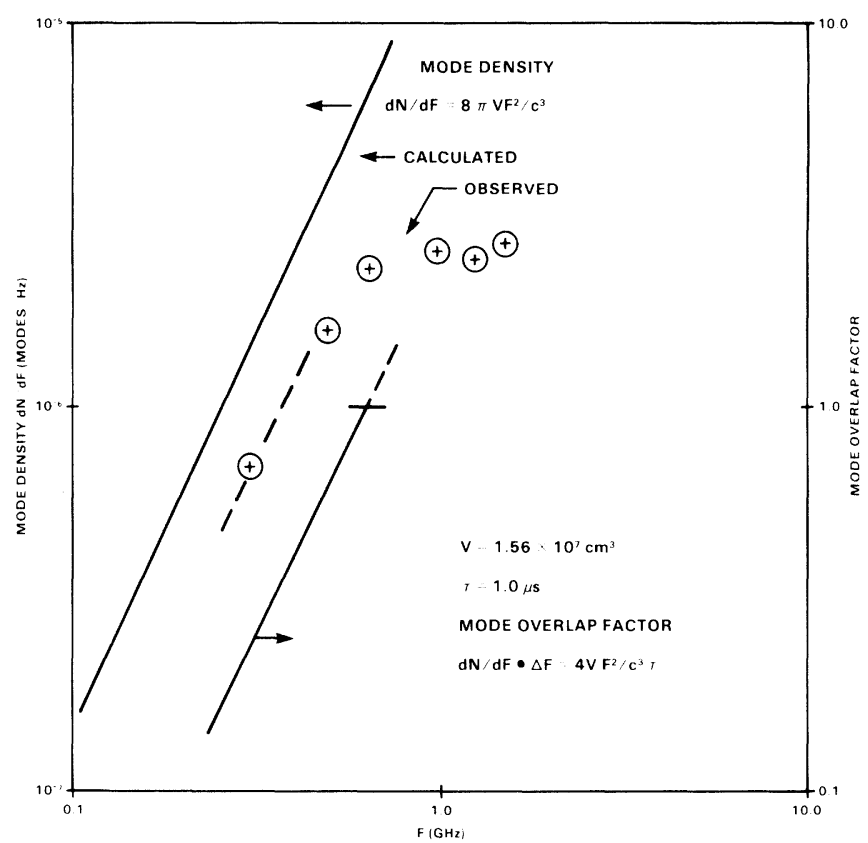

Fig. 10. Plot of calculated mode density, observed spectral peak density and calculated mode overlap factor for copper-lined MSC in use at N.S.W.C.

be worthwhile to examine it analytically in further work from a frequency-domain viewpoint.

The critical frequency $F_{\min }$ for mode overlap to occur may be evaluated by manipulation of (20), (21), and (22) assuming that a suitable overlap factor $(A)$ is unity. (Additional work may show that a slightly different value is more useful).

$$
F_{\left.\min \right|_{A=1}}=\sqrt{\frac{c^{3} \tau}{4 V}}=\frac{c}{2} \sqrt{M} \quad(\mathrm{~Hz}) .
$$

\section{Application of Results to MSC Design and Operation}

Several equations regarding chamber parameters have been presented:

$$
\begin{aligned}
\tau & =\frac{l}{2 \sqrt{2}} \sqrt{\frac{\sigma \mu_{0}}{\omega \hat{\mu}}} \\
M=c \tau / V & =\frac{1}{2 \sqrt{2}} \frac{1}{V^{2 / 3}} \sqrt{\frac{\sigma}{\omega \hat{\mu} \epsilon_{0}}} \\
F_{\min } & =\frac{c}{2} \sqrt{M} .
\end{aligned}
$$

Equations (14) and (15) provide upper limits to possible values of $\tau$ or $M$. Losses will, invariably, be greater than those implied by the wall loss terms. It may be pleasing to note that the calibration factor, which has units of inverse area, does depend upon the chamber surface area $\left(V^{2 / 3}\right)$. The term $\sqrt{\sigma / \hat{\mu} \epsilon_{0}}$ in (15) might be recognized as the inverse of the transmission factor $T(1+\Gamma)$ for field transmission into a metallic wall, which also appears in (13) defining $n$. Thus the calibration factor varies with the 
number of "bounces" a wave must make to drop to $1 / e$ of its initial value and inversely with chamber surface area.

The equations provide some guidance on chamber size considerations. A large chamber will tend to have a long $\tau$ and a lower value for $M$. However, large size does not automatically translate to low cutoff frequency $\left(F_{\min }\right)$, except insofar as large-size leads to lower $M$. If low-loss construction is used, this raising $M, F_{(\min )}$ will also increase.

Other factors, not discussed here, may also become important in determining $F_{\text {min }}$. Paddle wheel design or construction may provide an alternate limit. At sufficiently low frequencies, MSC losses may also be determined by feed structure properties, which would limit $M$ to about $\left(4 \pi / \lambda^{2}\right)$. The relationship between $M$ and $\tau$ is significant because of the direct trade-off between MSC response time and calibration factor, or $Q$, as described earlier. This relationship also implies that one can determine the powerdensity in an MSC simply by measuring the relaxation time and then measuring the net power injected into the chamber. It is not necessary to use a calibrated reference antenna or field probes.

Finally, the definitions of calibration factor (1), $Q$, and (5) can be compared with similar results derived [5] and used by other workers [6]. An alternate definition of $Q$ has been given as

$$
Q=16 \pi^{2} \frac{V}{\lambda^{3}} \frac{P_{R}}{P_{T}}
$$

where $P_{R}$ and $P_{T}$ are received and transmitted power levels. With a transmitted power level of $P_{T}$ and $\langle P D\rangle_{\text {eff }}$ given by (5):

$$
\langle P D\rangle_{\mathrm{eff}}=\frac{8 \pi}{\lambda^{2}} P_{R} \quad\left(\text { watt } / \mathrm{cm}^{2}\right)
$$

a reference antenna would receive a power level

$$
P_{R}=\langle P D\rangle_{\mathrm{eff}} \cdot \mathrm{A}_{\mathrm{eff}} \text { (watt) }
$$

consistent with (24) and (25) if $A_{\text {eff }}$ is given by

$$
A_{\text {eff }}=\frac{1}{2} \cdot \frac{\lambda^{2}}{4 \pi} .
$$

One would attribute (27) to a matched isotropic antenna with a $3-\mathrm{dB}$ polarization loss. Thus the formulations yielding (5) and (24) are apparently consistent.

\section{Summary}

In this paper, single-mode cavity theory and an ensemble averaging technique are combined to show the connection between MSC calibration factor and relaxation time. This allows one to deduce fields present in a chamber solely from a net power measurement and a transient response measurement with no need to measure fields directly.

Also, scaling laws are developed which show the relation between chamber size, wall loss, relaxation time, and minimum operating frequency. It is shown that minimum operating frequency depends directly on a chamber calibration factor rather than chamber size per se, thus leading to the conclusion that large chamber size may not always be required.

\section{ACKNOWLEDGMENT}

The author wishes to thank C. E. Gallaher and W. S. Lenzi for continued management support. The author is indebted to numerous individuals in Naval Surface Weapons Center (NSWC) branches, H22 and F11 for technical assistance and particularly to R. I. Gray of NSWC and M. Crawford of National Bureau of Standards for ideas gained in many long technical discussions.

\section{REFERENCES}

[1] B. H. Liu, D. C. Chang, and M. T. Ma, "Design considerations of reverberating chambers for electromagnetic interference measurements," in Symposium Record, IEEE Symposium on EMC, p. 508.

[2] J. D. Jackson, Classical Electrodynamics. New York: Wiley, 1962, p. 256.

[3] Y. W. Lee, Statical Theory of Comunication. New York: Wiley, 1960, p. 171.

[4] John L. Bean and Richard A. Hall,"Electromagnetic susceptibility measurements using a mode stirred chamber," in Record of 1978 IEEE Symposium on Electromagnetic Compatibility, pp. 143-149, June 1978.

[5] P. Corona, G. Latmiral, and E. Paolini, "Performance and analysis of a reverberating enclosure with variable geometry," IEEE Trans. Electromagn. Compat., vol. EMC-22, p. 2, Feb. 1980.

[6] M. L. Crawford and G. H. Koepke, "Operational consideration of a reverberation chamber for EMC immunity measurements-Some experimental results," presented at the IEEE Symp. on EMC, San Antonio, TX, Apr. 24-26, 1984. 